\title{
Fire Image Detection System based on SOA
}

\author{
HUANG Tongmiao ${ }^{1, a}$, BI Zhenbo ${ }^{1,2, b}$, GU Shenming ${ }^{1,2, c}$, \\ YE Jun ${ }^{1, d}$, PENG Shuyou ${ }^{1, e}$, XU Jianzhong ${ }^{1, a}$ \\ ${ }^{1}$ School of Mathematics, Physics \& Information Science, Zhejiang Ocean University, \\ Zhoushan, China 316022
}

${ }^{2}$ Key Laboratory of Oceanographic Big Data Mining \& Application of Zhejiang Province, Zhoushan, China 316022

\begin{abstract}
a719829416@qq.com, bbzb136@sina.com, 417119303@qq.com, dye1114126929@qq.com, e779751842@qq.com
\end{abstract}

\begin{abstract}
Key words: SOA, Digital image processing, fire image detection, Web Service
Abstract. Fire image detection is a kind of important means of early prevention of fire, which has important application value in the important energy base in the island, harbor or other large space. The current fire image detection is focus on the feature extraction, the concrete algorithm, etc., but fire detection system generality is not high. Aiming at this problem, this paper proposes a fire image detection scheme based on service oriented architecture (SOA). With the help of Web Service and workflow technology, the solution, to some extent, solves the difficult problem that fire image detection is not high for a long time, and it has important research value.
\end{abstract}

\section{Introduction}

Fire image detection is an important way to fire early prevention, which has an important application value in important energy base in the island, harbor and other large space. The detection technology includes three methods: smoke, fire, smoke and flame with the combination of fire detection. The detection method is based on the characteristics of the specific monitoring scenario and potential interference sources, uses static characteristics and dynamic characteristics of image performance of smoke or fire to detect fire. Now there are many different kinds of algorithm for smoke or flame detection [1] [2], they play an important role in fire detection in the early fire, but they are focus on the feature extraction, specific algorithm, etc. In general place of fire detection, the flexibility or universality of the detection systems based on the algorithms is not high. Analyzing the cause, we think the systems are lack of coordination and cohesion in the fire feature extraction and integration of each link, including other links. Thus they makes the function of the system single, not according to the actual to make the right fire detection decisions properly, which makes application range of the systems very limited.

SOA (Service-Oriented Architecture) is a kind of service-oriented architecture, this structure is a component model, it links applications' different functional units (called services) through well-defined interfaces and contracts between these services. The interface is defined in a neutral way, and is independent of the hardware platform, operating system and programming language which can be used to implement the services, and the services based on the architecture building in the variety of systems can use a unified and general way to interact. Web Service is a kind of implementation method of SOA, which can be visited by an open protocol normally. Fire Image detection based on SOA can implemented through Web Service, the functions in the process of the original fire detection are made into many web services, then detection tasks under different detection conditions are implemented as multiple processes through service portfolio based on 
workflow. The processes improve general usage of fire detection through the dynamic invocation of workflow engine.

Therefore, in the following, the research status of domestic and foreign related the research is summarized firstly, and then on the basis of an overview of Web Service and workflow, service design of fire image detection based on the Web Service and service composition based on workflow are studied, and finally the fire detection processes are tested through the workflow engine designed by us, which is used to verify the validity of the proposed method.

\section{Related research}

At present, there are a lot of applications based on SOA, but the applications related to fire image detection based on the of digital image processing technology are very few, fire image detection system based on Web Service was studied in the paper [4], the design and data processing of fire detection service were discussed On the basis of building the system architecture. For video or image processing, Hemalatha $\mathrm{T}$ et al [5] presented a distributed image processing system based on SOA, and the various functions of each stage were provided by Web services in the image processing. Giro-i-Nieto $\mathrm{X}$ et al [6] studied the implementation of the web service architecture for content-based image retrieval. A medical image processing platform which is used to assistant diagnosis based on SOA architecture is proposed in the paper [7]. With the REST service technology and service-oriented architecture, the geography stereoscopic video web services were implemented in the paper[8]; Remote sensing image processing system based on Web service, workflow technology, and series standards of OGC Web Services was implemented in web environment[9]; a video monitoring integrated service platform based on SOA architecture and cloud computing technology was design [10]; LIANG Yi et al[11] presented an image processing solutions for mobile devices through Web Service, which solved the problem of the limited computing resources on the mobile device through the traditional image processing methods. These research involved multiple areas of the image application, different from traditional image application platform, they made full use of the advantage of building application based on SOA, the advantage includes loose coupling, platform-independent, distributed and strong flexibility, which would provide important theoretical and technical guidance for the research of this paper.

\section{Design and implementation of fire image detection system based on SOA}

General framework of the system. Fire image detection system based on SOA is mainly composed of three functional layers, as shown in Fig.1.

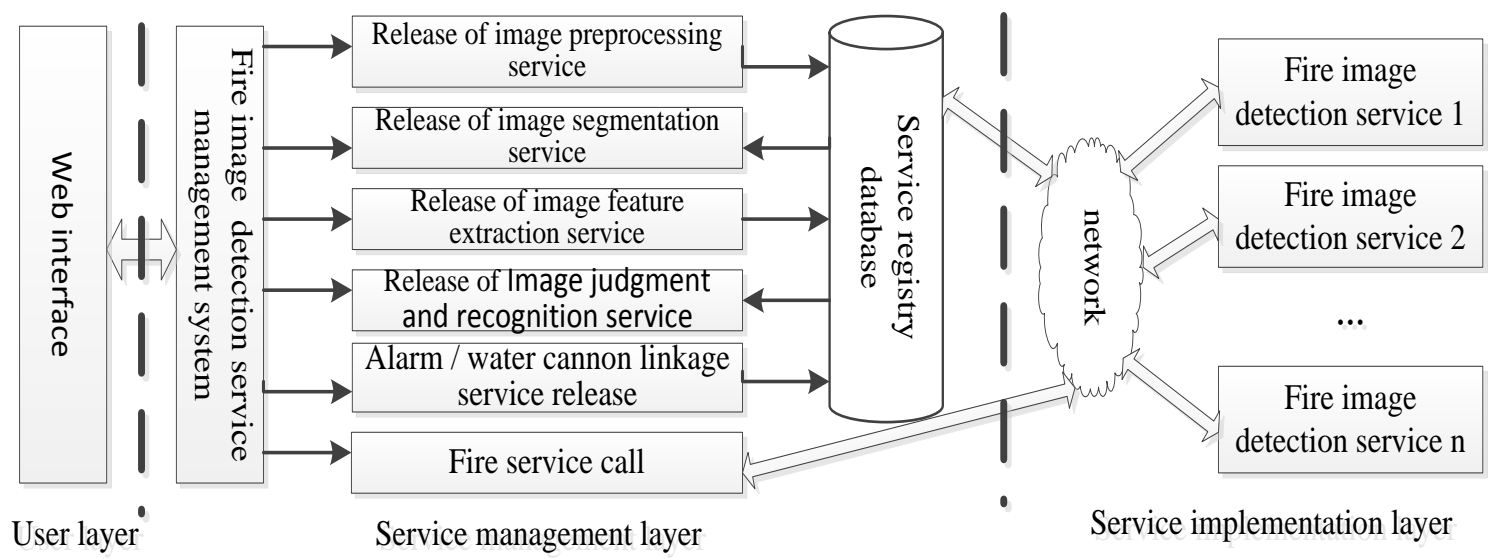

Fig. 1 the architecture of fire image detection based on SOA 
User Layer: it is a client of fir image detection system based on SOA. Users use the existing terminal equipment to achieve the start of fire detection and system settings through the web interface.

Service Management Layer: it is mainly includes service release, discovery, invocation and management of fir image detection system, which ensures the quality of fire detection service.

Service Realization Layer: it is the realization of specific fire image processing services in fir image detection system based on Web Service. A fire detection service distributed in each location on the network is equivalent to one "black box" which provides a variety of methods of the interface, but hides the details of its implementation, which will contribute to service discovery and service composition of the application of fire detection.

System development steps. Web Service is an implementation of SOA. In the specific development, the system will be based on Web Service to be implemented. Web service can be regarded as a kind of object which is deployed on Web. The development of fire image detection system by Web service is different from the development of traditional software system, which involves Web service deployment, discovery and the process of invocation. The Development steps are as follows:

System building stage: it mainly complete the definition and description of the service provider's service interface. At this stage, five types of functional interface will be mainly achieved, they are image preprocessing service interface, image segmentation service interface, fire feature extraction service interface, fire determination and alarm/water cannon Linkage saves service interface, and so on.

Service deployment stage: this stage is used for completing to release service interface and service description in service registry center, namely registering in UDDI. UDDI defines the Web Service interface's storage path which described by WSDL and haven been embedded in the Microsoft.NET development environment. The address of the Web service is registered to UDDI, that users can visit the address to find web service interface of the fire image processing which described by WSDL. Users finally create the WSDL document into a Tmodel structure to produce a TModelKey (with Web service binding), registered in http: //uddi.microsoft.com/.

Discovery and invocation: realizing discovery and invocation of web services, the service discovery in this system mainly used the service discovery algorithm[12] based on keyword, which get specific service entry address and service description information from service registry center through keyword matching. Then, combined with user participation, with the help of workflow technology, the specific business process of fire detection was built (Corresponding to a specific fire detection application system).

Implementation of the specific system. Work is divide work into well-definition tasks and roles which are performed according to the specific rules and processes, thus workflow is achieved [13]. In the workflow standard field of Web service, there have been some certain characteristics and similar languages at present, such as XLANG、WSFL and BPEL4WS, etc. BPEL4WS is a product of combining the advantages of XLANG and WSFL (supporting graph oriented process and supporting structured construction of a process), which forms a very natural way to support the implementation of various types of business process specification. Describing web service behavior and control logic in business process interactions through BPEL can integrate isolated, stateless web services to play the full potential of web service technology as fire detection system. Web services view based on BPEL is shown in Fig. 2. The business process is defined by the execution order of a set of Web service operations by BPEL, including the service type definition and the business process definition. The former is some type definition used by web services (such as data type, 
message type, port type, type of partner link, etc.); the latter is used to define the business process itself (including variables, correlation, activities, partner links and endpoint reference, etc). Invoke is used to invoke the operation of a Web service, receive is used to receive messages of foreign resources, reply is used to respond to external resources, etc.

With the developing of workflow, workflow modeling techniques and tools, it is conducive to decompose a complex problem and rebuild Processing process [7]. The workflow technology achieves the separation of application logic and process logic [7], and the combination of workflow and Web Service will make the application logic and process logic of fire image detection be effective unification, which provides the technical basis for realizing complex fire detection in web

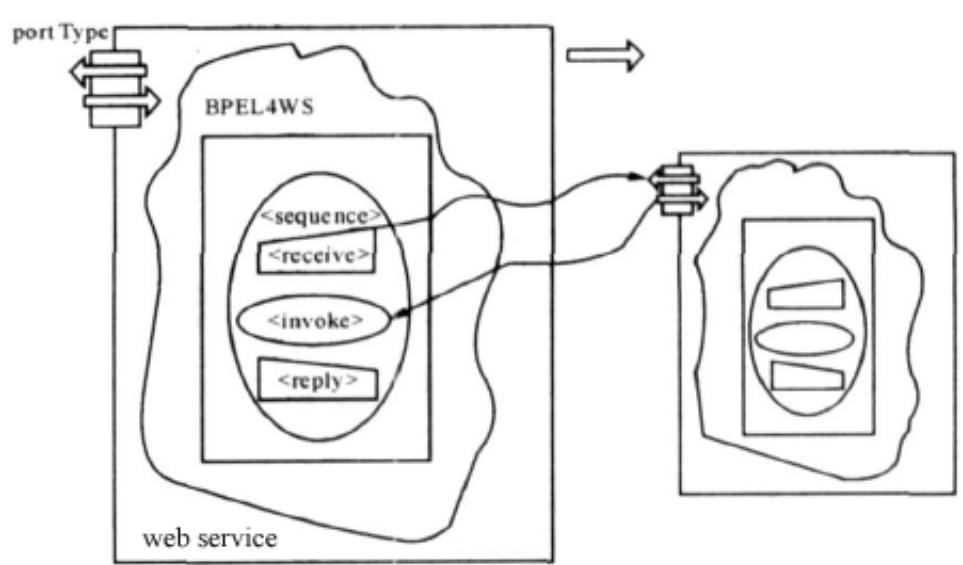

Fig. 2 the implemented Web service view based on BPEL mode, and provides the conditions for the effective solution of universality of fire detection. Methods are as follows:

1) In a specific condition or environment, Fire detection task is essentially a process of fire detection which can be constructed by workflow technology.

2) The difference between the different processes lies in the change (diurnal variation, the change of illumination in different time of the same day and other change). Therefore, a fire detection system should have different processes.

3) The construction a specific process is a process carrying out Web service discovery and combination by certain UDDI and Web service discovery is realized by matching the interface and functionality based on keywords. These different Web services respectively represent different application logic in the process of fire detection.

4) The fire detection processes in the different conditions constitute a powerful universal adaptive fire detection system that can be used to detect fire through the unified scheduling of workflow engine.

For the above realization methods, general requirements are that web services registered in UDDI must be enough, which let Web service consumer (here refers to the developers of fire image detection system) can choose what they need, so as to create function module meeting different fire detection tasks or processes through service composition technology based on workflow. In addition, the types of services (rich interface and function) should meet the needs of fire detection in different conditions (preprocessing, image segmentation, feature extraction, intelligent fire recognition and alarm). In reality, these services in commercial or free way are generally provided by third party service providers. As a study, this article has only studied smoke image fire detection in a large space environment. The general process of the fire detection system is shown in Fig.3. The query interface is provided for the purpose of fire video viewing or playback which is based on the traditional date or keywords retrieval and content-based query. The fire detection is the core of this paper, so the fire image retrieval is omitted here (this content is not included in the test of this study).

In fact, fire image detection, whether for smoke or flame or their combination, all include video preprocessing, feature extraction, intelligent analysis, fire judgment and identification, alarm, water 
gun linkage and fire video database storage, etc. Among them, fire image feature extraction includes extraction of smoke and flame features, multi feature fusion, multi-dimensional feature classification and recognition processing. The whole process of fire detection involves multiple modules in order and coordination, it generally show a clear task flow. The flow difference in different detection conditions is that the interface parameters of similar function services of each node are different (having different service implementations). Thus, the coexistence of the different detection processes has been formed (unified scheduling by workflow engine which is responsible for parsing application requirements and makes corresponding processing, but only one process at a time is booted) in order to cope with fire detection (through process reconstruction) in different conditions (user participation system parameter settings or environmental change found by system can be self-adjustment).

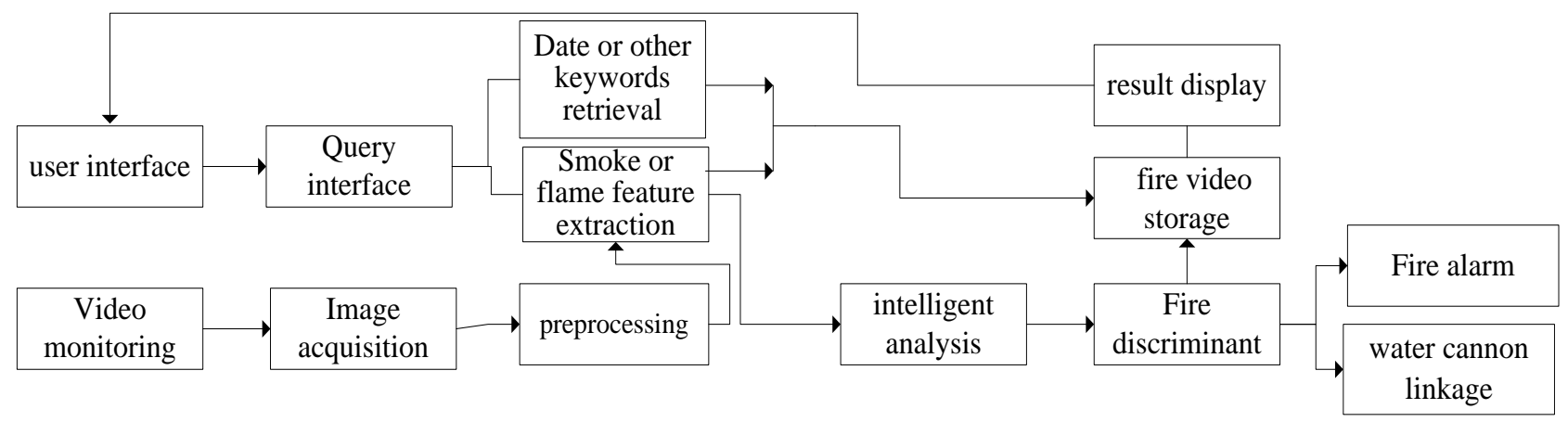

Fig.3 Fire image detection system

The LAN test environment of system is made up of 3 PCs with same configuration. They respectively act as service requesters, service registry center, and service provider, and Windows server 2003 is installed in the service provider machine. Based on B/S architecture, the server-side is implemented by IIS and SQL Server 2005, and user interface is implemented by ASP.NET, JavaScript and HTML, and we are assisted to complete a variety of web services discovery through using API function provided by UDDI SDK libraries in net. When the system is designed, some indicators are exposed as far as possible through parametric design. The final implementation is shown in Fig.4, figure (a) is the generation interface of fire detection process, and figure (b) is the corresponding flow operation interface. In tests, we have shot and got ignition video in the large space of $4.5 \mathrm{~m} * 3 \mathrm{M} * 30 \mathrm{~m}$, The height of the camera installation is greater than or equal to $4 \mathrm{~m}$, the ignition material and the interference light source refer to the standard of the special type fire detector. In system testing and running, parameters can be set at different times, system can generate different workflow to cope with fire detection in different conditions, the test shows that system has reached the expected goal.

In short, there are many factors that cannot be expected in fire image detection, Any fire detection system is not likely to take all factors into consideration. In order to guarantee the adaptability of the fire detection system, the system provides many options which are set by the user according to the actual environment and generate specific detection process with the help of the workflow, thus the system effectively deal with the different situations, which improves the generality of fire detection system.

\section{Conclusions}

This paper is aimed at the problem of poor flexibility or low commonality of previous fire image detection, a solution of fire image detection based on SOA is proposed. Fire image detection prototype system based on SOA by using Windows communication foundation (WCF) and 
windows Workflow Foundation (WF) is implemented. Experiments show that the solution with web service and workflow technology in a certain extent solved the long-standing fire image detection problem in general low, which provides the idea of solving the problem of fire detection in all areas of the next step and has important research value. But looking from the actual test results, this study still need to do further improvement and optimization in automatic environment judgment and other relevant to the implementation of aspects.

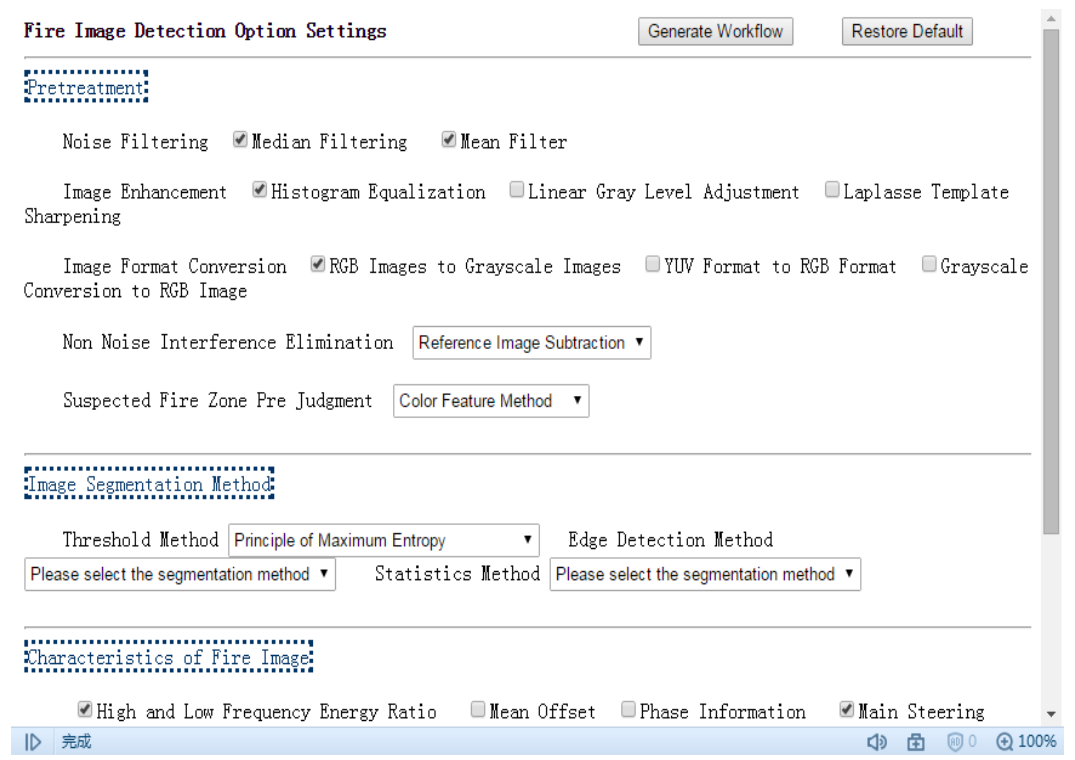

(a)

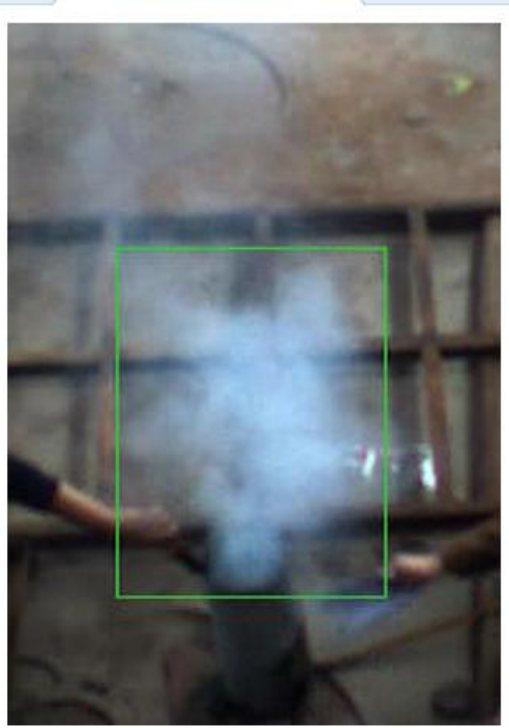

(b)

Fig.4 Test of the system

\section{Acknowledgements}

This work was financially supported by Zhejiang Province College Students' Science and Technology Innovation Activity Plan (New Talent Plan) Projects (2015R411034).

\section{Reference}

[1] Yan HU, Wang H, Qin W, et al. Fire image features selection and recognition based on rough set[J]. Journal of Computer Applications, 2013, 33(3):704-707.

[2] YAO Tai-wei, WANG Hui-qin, HU Yan. Fire Smoke Detection Based on Wavelet Transform and Sparse Optical Flow Method [J].Computer Engineering, 2012, 38(6):204-206.

[3] Xiao F, Zhang W H, Wang D H. Overview of workflow technology in scientific process[J]. Application Research of Computers, 2011, 28(11):4013-4019.

[4] Zhen bo B I, Wang H Q, Ying L U, et al. Research of fire image detection based on cloud computing [J]. Fire Science \& Technology, 2013.

[5] Hemalatha T, Athisha G, Jeyanthi S. Dynamic web service based image processing system[C]//Advanced Computing and Communications, 2008. ADCOM 2008. 16th International Conference on. IEEE, 2008: 323-328.

[6] Giro-i-Nieto X, Ventura C, Pont-Tuset J, et al. System architecture of a web service for content-based image retrieval[C]/Proceedings of the ACM International Conference on Image and Video Retrieval. ACM, 2010: 358-365. 
[7] Todica V, Vaida M F. SOA-based medical image processing platform[C]//Automation, Quality and Testing, Robotics, 2008. AQTR 2008. IEEE International Conference on. IEEE, 2008, 1: 398-403.

[8] Han Z, Kong Y, Wang Z, et al. Geographic Stereo Video Web Services: Framework Design and Interface Development[J]. Geo-information Science, 2013, 15(1):97-105.

[9] Zhang D R, Le Y U, Deng C, et al. OGC WPS-based remote sensing image processing in Web environment[J]. Journal of Zhejiang University, 2008, 42(7):1184-1188.

[10]Han H W, De yu Q I, Feng B. Video surveillance integrated service platform based on cloud computing technologies[J]. Computer Engineering \& Design, 2013, 34(5):1657-1662.

[11]Liang Y, Xiao Y Y, Huang J, et al. Image processing technology oriented mobile devices based on Web services[J]. Computer Engineering \& Applications, 2009, 45(35):62-63.

[12]Klusch M, Fries B, Sycara K. Automated semantic web service discovery with OWLS-MX[C]//Proceedings of the fifth international joint conference on Autonomous agents and multiagent systems. ACM, 2006: 915-922.

[13] Van Der Aalst W, Van Hee K M. Workflow management: models, methods, and systems[M]. MIT press, 2004. 